\title{
Properties of Periodic Arrays of Symmetric Complementary Structures and Their Application to Grid Amplifiers
}

\author{
Alina Moussessian, Member, IEEE, James J. Rosenberg, Member, IEEE, and David B. Rutledge, Fellow, IEEE
}

\begin{abstract}
Deschamps' theorem for $n$-terminal complementary structures is reviewed. An extension to Deschamps' theorem for a class of three-terminal bounded structures with one axis of symmetry is presented. It is shown that, for these structures, a simple relationship between the impedances of the odd mode of the original structure and the admittances of the even mode of the complementary structure exists, and that these modes are orthogonal. Using this, a self-complementary grid amplifier is designed and the measured results are presented.
\end{abstract}

Index Terms- Grid amplifiers, quasi-optics, self-complementary.

\section{Motivation}

Q UASI-OPTICAL grid amplifiers using differential-pair transistors have been used to combine the output power of many solid-state devices in free space, eliminating the losses associated with waveguide or transmission-line combiners [1], [2]. To the first order, the unit cell of a grid determines the driving-point impedance seen by each device, while power scales with the grid area. This allows the reduction of the problem of the entire grid to that of a single unit cell. The symmetry of some grids allows boundary conditions to be imposed on a unit cell, thus reducing the problem of solving for the cell to that of solving for a waveguide representing the cell [3]. Previous grid-array amplifiers have used crosseddipole antennas for input and output. The current distributions on these dipole antennas do not permit a simple solution for fields in the equivalent waveguide. Consequently, previous grid amplifiers have been modeled using an approximate transmission-line equivalent-circuit model [2]. The crosseddipole construction has the additional drawback that it requires the use of differential pairs of transistors which must be specially fabricated for the application.

A unit-cell configuration that can be more readily modeled would be desirable, as would a configuration that utilizes a single transistor per unit cell while maintaining cross-polarized

Manuscript received March 20, 1998; revised July 27, 1998. This work was supported by the Physical Optics Corporation and by the U.S. Army Research Office.

A. Moussessian is with the Jet Propulsion Laboratory, Pasadena, CA 911098099 USA.

J. J. Rosenberg is with the Department of Engineering, Harvey Mudd College, Claremont, CA 91711 USA.

D. B. Rutledge is with the Department of Electrical Engineering, California Institute of Technology, Pasadena, CA 91125 USA.

Publisher Item Identifier S 0018-9480(98)08337-9.

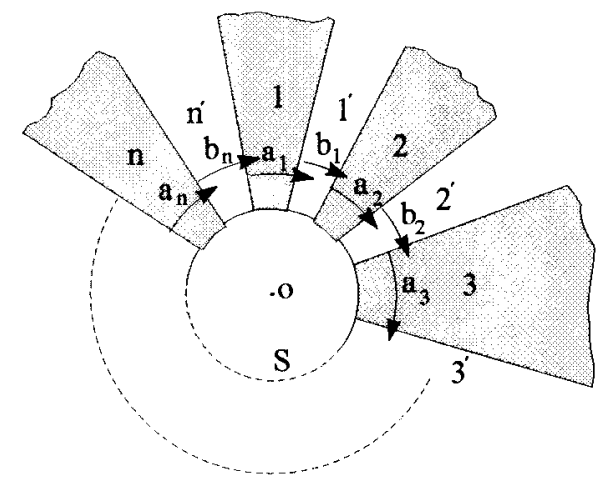

Fig. 1. An $n$-terminal structure.

input and output. Periodic arrays of self-complementary symmetrical structures offer such an alternative configuration.

\section{Deschamps' TheOREM}

Using Babinet's principle, Booker presented a simple relationship between the impedances of two one-port planar complementary structures [4]. In 1959, Deschamps presented the impedance properties of multiterminal complementary planar structures [5], a generalization to Booker's equation. Fig. 1 shows an $n$-terminal structure. Assume that a source inside $S$ is connected to some or all of the terminals. The sphere $S$ is small compared to the wavelength of operation. Assume that $F=(E, H)$ is a field solution for this structure. In other words, $F$ is a field produced about this structure by some configuration of sources inside $S$.

Now consider the complement of the structure in Fig. 1, obtained by replacing the metal parts of the original structure with free space and the apertures with metal. Deschamps showed that an acceptable solution for this structure is the dual of $F . F^{\prime}$ is defined to be the dual field

$$
F^{\prime}=\left(E^{\prime}, H^{\prime}\right)=\left(-\eta_{o} H, \frac{1}{\eta_{o}} E\right)
$$

where $\eta_{0}=377 \Omega$ is the characteristic impedance of free space.

To show that $F^{\prime}$ is a solution to the complement of the structure, first consider the uniqueness theorem. For linear isotropic media, specification of the boundary conditions is sufficient to specify fields uniquely within the region. Therefore, two problems with the same boundary conditions will have identical 


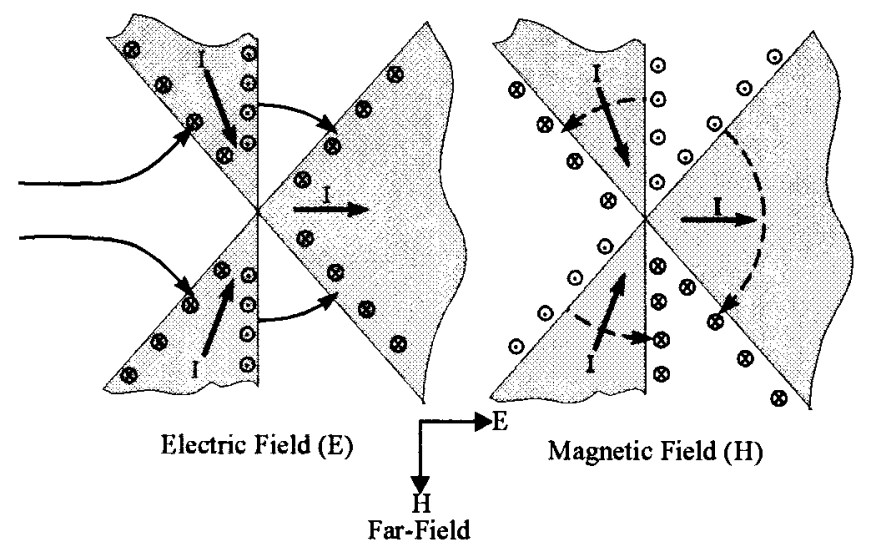

(a)

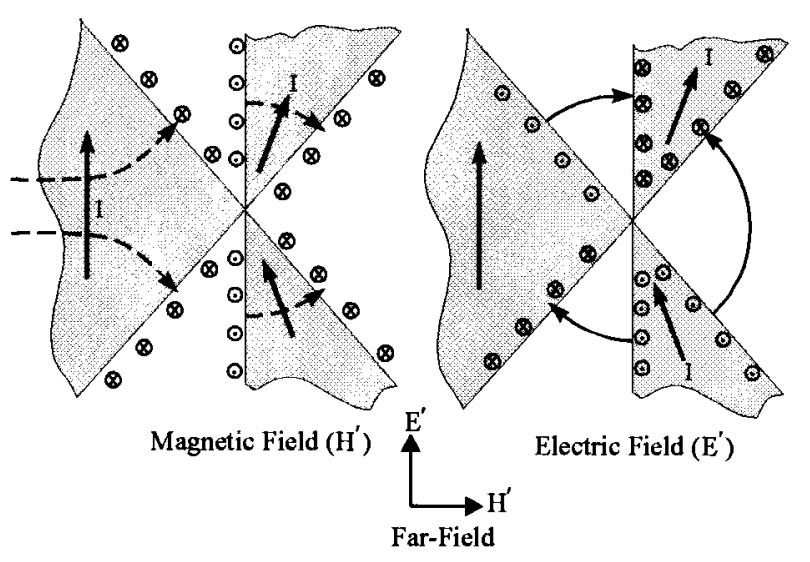

(b)

Fig. 2. (a) Electric and magnetic fields for an arbitrary structure. (b) The electromagnetic fields for the complement of the structure in (a). The fields in (b) are the duals of the fields in (a).

solutions. Consider the structure of Fig. 2(a) with the assumed field solution $F$. Continuity of the tangential electric field at a boundary requires that the surface tangential electric field be zero just outside the metal surface. Therefore, the $E$-fields at the plane of the structure approach the metal normal to its boundary and, just above the structure, the $E$-fields are normal to the plane of the structure. The discontinuity in tangential magnetic field requires that the tangential magnetic field be perpendicular to the direction of the surface current and equal to the surface current density. Therefore, the $H$-fields are parallel to the plane of the structure above and below it and, at the metal boundary, the fields cross the plane of the structure normal to the surface.

The electric and magnetic fields about this planar structure are shown in Fig. 2(a) for the original structure and in Fig. 2(b) for the complementary structure. Note that the boundary conditions for the $E$-fields in Fig. 2(a) are similar to the $H$-field boundary conditions in Fig. 2(b). The same similarity exists between the $H$-field boundary conditions in Fig. 2(a) and the negative of the $E$-field boundary conditions in Fig. 2(b). Therefore, the boundary conditions for the complement of the original structure are the duals of the original boundary conditions. It is clear that assuming $F$ is the field solution for Fig. 2(a), $F^{\prime}$ (dual of $F$ ) not only satisfies Maxwell's

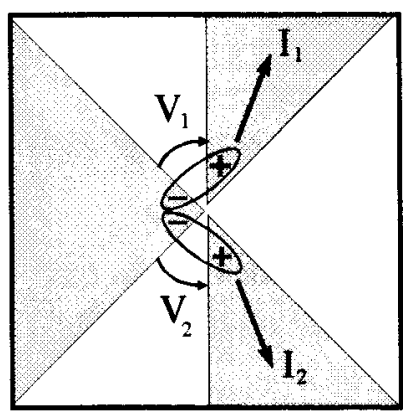

Fig. 3. Self-complementary two-port structure.

equations, but also satisfies the boundary conditions of the complementary structure shown in Fig. 2(b). Therefore, due to the uniqueness theorem, $F^{\prime}$ is the field solution for Fig. 2(b). In other words, the field solutions for the complement of a structure is equivalent to the field solution for the dual of that structure.

Deschamps showed that the voltages and currents of the complementary structure can be defined using the voltages and currents of the original structure [2]

$$
\begin{aligned}
I_{i}^{\prime} & =\frac{2}{\eta_{o}}\left(V_{i}-V_{i+1}\right), \quad i=l, \cdots, n \\
V_{i}^{\prime}-V_{i+1}^{\prime} & =-\frac{\eta_{o}}{2} I_{i+1}, \quad i=1, \cdots, n
\end{aligned}
$$

where $I_{i}$ is the current flowing into terminal $i$ and $V_{i}-V_{i+1}$ is the voltage difference between terminals $i$ and $i+1$ of Fig. 1. Similarly, $I_{i}^{\prime}$ is the current flowing into terminal $i^{\prime}$ of the complementary structure shown in Fig. 1 , and $V_{i}^{\prime}-V_{i+1}^{\prime}$ is the voltage difference between terminals $i^{\prime}$ and $i^{\prime}+1$ of this structure. By convention, $n+1$ is equal to 1 .

\section{An Extension to Deschamps' TheOREM}

Here, we discuss an extension to Deschamps' theorem for a symmetrical three-terminal (two-port) rectangular structure, bounded with electric and magnetic walls, such as shown in Fig. 3. Adjacent walls are of opposite type. As will be discussed later, such a boundary condition might arise from a periodic array. The symmetry we refer to throughout this discussion is with respect to a central axis parallel to either boundary. For this discussion, we will define an even-mode voltage $V_{e}$ and an odd-mode voltage $V_{o}$ in terms of the voltages $V_{1}$ and $V_{2}$ of Fig. 3 as follows:

$$
\begin{aligned}
& V_{e} \equiv \frac{V_{1}+V_{2}}{2} \\
& V_{o} \equiv V_{1}-V_{2} .
\end{aligned}
$$

Similarly, $V_{1}$ and $V_{2}$ can be expressed in terms of $V_{e}$ and $V_{o}$ as follows:

$$
\begin{aligned}
& V_{1}=V_{e}+\frac{V_{o}}{2} \\
& V_{2}=V_{e}-\frac{V_{o}}{2} .
\end{aligned}
$$

In the following discussion, an odd-mode excitation is defined as the mode where equal voltages with opposite polarity appear at ports $a_{o}$ and $b_{o}$ of the structure, as shown in 
Fig. 4(a), with $V_{e}=0$. An even-mode excitation is defined as the mode where equal voltages with the same polarity appear at ports $a_{e}$ and $b_{e}$ of the structure, as shown in Fig. 4(b), with $V_{o}=0$. We will prove that the ratio of the impedances of the odd excitation of such a structure to the corresponding admittances of the even excitation of its complementary structure is constant and is equal to $\eta_{o}^{2} / 4$, where $\eta_{o}$ is the characteristic impedance of free space.

The results stated above apply to any symmetrical structure with previously described boundaries and its complement. In the case of a self-complementary structure, there are additional simplifications. A self-complementary structure is one which looks the same when the metal part is exchanged with the nonmetal part. For a self-complementary structure, the ratio of the impedances of the odd excitation of the structure to the admittances of the even excitation of the same structure is constant and is equal to $\eta_{o}^{2} / 4$.

Consider a source configuration at the terminals of the structure in Fig. 4(a) which results in an odd-mode excitation. At low frequencies where the structure only supports a single TEM mode, this odd-mode excitation generates a vertically polarized electric field $\left(E_{V}\right)$ at the far-field. The complement of this structure is shown in Fig. 4(b). As discussed earlier, boundary conditions for the complement of a planar structure are the duals of the boundary conditions for the original structure. To use Deschamps' theorem for a bounded structure, the $E$ - and $H$-walls of the original structure must be changed to their duals when complementing the structure. Since the dual of an $H$-wall is an $E$-wall and vice versa, this is equivalent to interchanging $E$ - and $H$-walls. The field $F^{\prime}=$ $\left(E^{\prime}, H^{\prime}\right)$ in Fig. 4(b) has to be the dual of the field $F=$ $(E, H)$ in Fig. 4(a), therefore, the far-field electric field for the new structure is horizontally polarized $\left(E_{H}\right)$ and the excitation generating it is an even-mode excitation, as shown in Fig. 4(b).

Bounding a planar structure with electric and magnetic walls is equivalent to analyzing this structure inside a waveguide with the same $E$ - and $H$-walls [3]. Fig. 5(a) and (b) shows the waveguide representation of the structures in Fig. 4(a) and (b). We define port 1 of Fig. 5(a) as the series combination of ports $a_{o}$ and $b_{o}$ in Fig. 4(a), and port 1 in Fig. 5(b) as the parallel combination of ports $a_{e}$ and $b_{e}$ in Fig. 4(b). Two hollow dots in Fig. 5(a) and two ovals in Fig. 5(b) show port 1. For the remainder of this discussion, we will refer to port 1 of Fig. 5(a) and (b) as the odd and even-mode device ports. The waveguides in Fig. 5(a) and (b) extend to both sides of the structures. However, due to reciprocity and to simplify the analysis of this three-port structure, these parallel ports are combined into one port. The new port [port 2 in Fig. 5(a) and (b)] has half the characteristic impedance of free space. We will refer to the parallel combination of these two ports as the waveguide port. The purpose is to find equations relating the equivalent circuits of the two-port waveguide structures shown in Fig. 5(a) and (b).

Consider the odd-mode excitation of this symmetrical structure inside the waveguide, [see Fig. 5(a)]. This odd-mode excitation at the device port generates a vertically polarized electric field $\left(E_{V}\right)$ at the waveguide port. Assume that this two-port structure can be represented by a T-equivalent circuit.

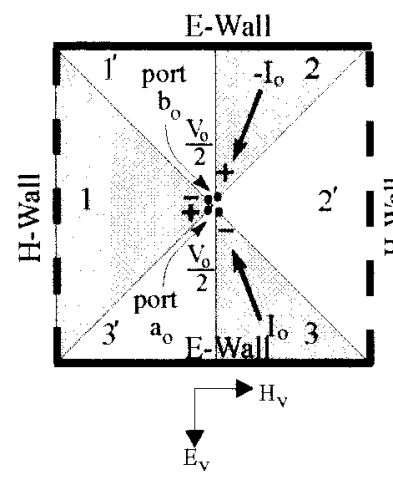

(a)

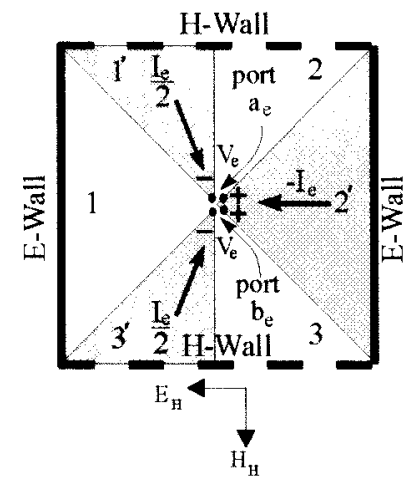

(b)
Fig. 4. (a) The odd excitation of a bounded symmetrical structure. (b) Even excitation of the complement of (a). The ports are marked with black dots.

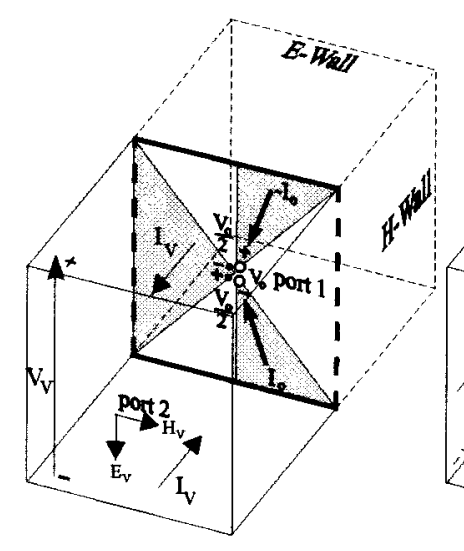

(a)

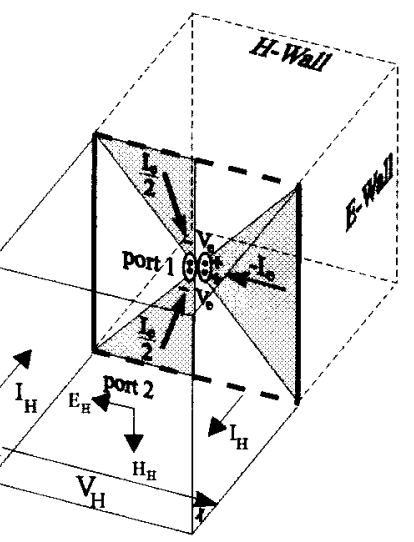

(b)
Fig. 5. Waveguide representation of Fig. 4. (a) Port 1 is the series combination of the ports in Fig. 4(a), shown by two hollow dots. (b) Port 1 is the parallel combination of the ports in Fig. 4(b), shown by two ovals. Port 2 in (a) and (b) is the parallel combination of the two ports of the waveguide.

The components of this T-circuit are $Z_{o i}(i=1,2,3)$. The $Z$-parameter matrix elements relating the odd-mode excitation of the device port to the vertically polarized field at the waveguide port are $Z_{\mathrm{ojk}}(j=1,2 ; k=1,2)$. The T-circuit components and the $Z$-parameters matrix elements are related, as shown in Fig. 6(a). This T-equivalent circuit relates the odd-mode voltage and current $\left(V_{o}\right.$ and $\left.I_{o}\right)$ at the device port to the waveguide port voltage and current $\left(V_{V}\right.$ and $\left.I_{V}\right)$. These voltages and currents are shown in Fig. 5(a).

Now consider the even-mode excitation of the complement of the structure in Fig. 5(a) which generates fields with horizontally polarized $E$-field $\left(E_{H}\right)$ at the waveguide port, as shown in Fig. 5(b). A П-equivalent circuit, as shown in Fig. 6(b), is used to represent the new structure. The components of this $\Pi$-circuit are $Y_{e i}(i=1,2,3)$. The $Y$ parameter matrix elements relating the even-mode excitation of the device port to the horizontally polarized field at the waveguide port are $Y_{\mathrm{ejk}}(j=1,2 ; k=1,2)$. The $\Pi$-circuit components and the $Y$-parameters matrix elements are related, as shown in Fig. 6(b). This $\Pi$-circuit relates the device port even-mode voltage and current $\left(V_{e}\right.$ and $\left.I_{e}\right)$ to the voltage and current at the waveguide port $\left(V_{H}\right.$ and $\left.I_{H}\right)$, as shown in Fig. 5(b). 


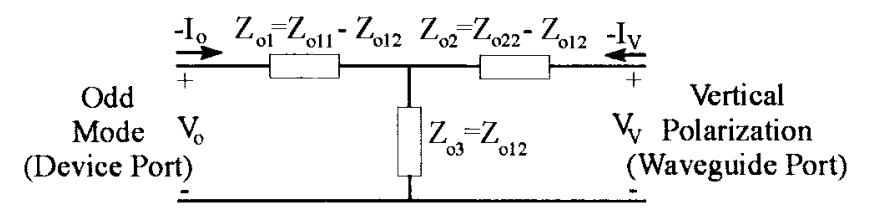

(a)

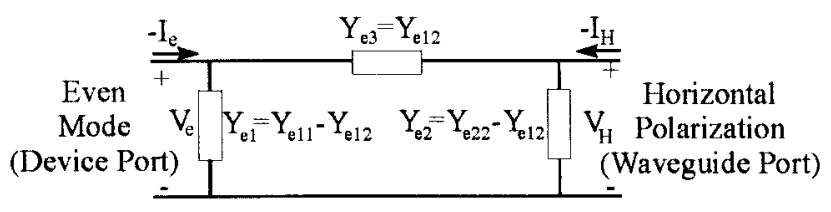

(b)

Fig. 6. (a) The T-equivalent circuit for the odd-mode excitation of the structure in Fig. 5(a). (b) The П-equivalent circuit for the even-mode excitation of the structure in Fig. 5(b). Due to reciprocity $Z_{\mathrm{o} 12}=Z_{\mathrm{o} 21}$ and $Y_{\mathrm{e} 12}=Y_{\mathrm{e} 21}$. have

Using (2) and (3) for the structures in Fig. 4(a) and (b), we

$$
\begin{aligned}
I_{o} & =-\frac{2}{\eta_{o}} V_{e} \\
V_{o} & =-\frac{\eta_{o}}{2} I_{e}
\end{aligned}
$$

where $I_{o}$ and $V_{o}$ are the odd-mode current and voltage, as shown in Fig. 4(a), and $I_{e}$ and $V_{e}$ are the even-mode current and voltage, as shown in Fig. 4(b).

Using (8) and (9),

$$
\frac{V_{o}}{I_{o}}=\frac{\eta_{o}^{2}}{4} \cdot \frac{I_{e}}{V_{e}} .
$$

Using Fig. 6, the ratio of the device-port impedance for the odd excitation [see Fig. 6(a)] to the device-port admittance for the even excitation [see Fig. 6(b)] when the waveguide ports are terminated to $\eta_{o} / 2 \Omega$ is

$$
\frac{V_{o} / I_{o}}{I_{e} / V_{e}}=\frac{Z_{\mathrm{o} 11}-\frac{Z_{\mathrm{o} 12}^{2}}{\eta_{o} / 2+Z_{\mathrm{o} 22}}}{Y_{\mathrm{e} 11}-\frac{Y_{\mathrm{e} 12}^{2}}{2 / \eta_{o}+Y_{\mathrm{e} 22}}}
$$

where $Z_{\mathrm{o} 11}, Z_{\mathrm{o} 12}$, and $Z_{\mathrm{o} 22}$ are the $Z$-parameters of the structure with odd-mode excitation and $Y_{\mathrm{e} 11}, Y_{\mathrm{e} 12}$, and $Y_{\mathrm{e} 22}$ are the $Y$-parameters of the complementary structure with even-mode excitation. Terminating the waveguide port to $\eta_{o} / 2 \Omega$ is equivalent to terminating the two ports of the waveguide in Fig. 5(a) or (b) to the characteristic impedance of free space.

Now consider shorting the device port in Fig. 5(a). In order for Fig. 5(b) to remain the complement of Fig. 5(a), the device port in Fig. 5(b) has to be an open. Fields at the waveguide port of Fig. 5(a) are related to the waveguide port fields of Fig. 5(b) by (1). We can derive

$$
\frac{\left.\frac{V_{V}}{I_{V}}\right|_{V_{o}=0}}{\left.\frac{I_{H}}{V_{H}}\right|_{I_{e}=0}}=\frac{\eta_{o}^{2}}{4}
$$

where $V_{V}$ and $V_{H}$ are the line integrals of $E_{V}$ and $E_{H}$ between the $E$-walls and $I_{V}$ and $I_{H}$ are twice the line integral of the $H_{V}$ and $H_{H}$ between the $H$-walls.

The same approach can be used when the device port in Fig. 5(b) is shorted. In this case, the device port in the complementary structure [see Fig. 5(a)] has to be an open circuit. Similarly, we can derive

$$
\frac{\left.\frac{V_{H}}{I_{H}}\right|_{V_{e}=0}}{\left.\frac{I_{V}}{V_{V}}\right|_{I_{o}=0}}=\frac{\eta_{o}^{2}}{4} .
$$

From the circuits in Fig. 6, we can also derive

$$
\begin{aligned}
& \frac{\left.\frac{V_{H}}{I_{H}}\right|_{V_{e}=0}}{\left.\frac{I_{V}}{V_{V}}\right|_{I_{o}=0}}=\frac{Z_{\mathrm{o} 22}}{Y_{\mathrm{e} 22}} \\
& \frac{\left.\frac{V_{V}}{I_{V}}\right|_{V_{o}=0}}{\left.\frac{I_{H}}{V_{H}}\right|_{I_{e}=0}}=\frac{Z_{\mathrm{o} 22}-Z_{\mathrm{o} 12}^{2} / Z_{o} 11}{Y_{\mathrm{e} 22}-Y_{\mathrm{e} 12}^{2} / Y_{\mathrm{e} 11}} .
\end{aligned}
$$

From (13) and (14),

$$
\frac{Z_{\mathrm{o} 22}}{Y_{\mathrm{e} 22}}=\eta_{o}^{2} / 4
$$

Solving for (10)-(12), (15), and (16), we can derive

$$
\begin{aligned}
& \frac{Z_{\mathrm{o} 12}}{Y_{\mathrm{e} 12}}=\eta_{o}^{2} / 4 \\
& \frac{Z_{\mathrm{o} 11}}{Y_{\mathrm{e} 11}}=\eta_{o}^{2} / 4 .
\end{aligned}
$$

To summarize, (16)-(18) simply indicate that for the ports shown in Fig. 6, the $Z$-parameters for the odd-mode excitation are related to the $Y$-parameters of the complementary structure with even-mode excitation by

$$
\frac{Z_{\mathrm{oij}}}{Y_{\mathrm{eij}}}=\eta_{o}^{2} / 4, \quad i=1,2, \quad j=1,2 .
$$

Using (19) and Fig. 6, the impedances of the T-equivalent circuit components $\left(Z_{\circ 1}, Z_{\circ 2}, Z_{\circ 3}\right)$ for the odd-mode excitation and the admittances of the $\Pi$-circuit components $\left(Y_{o 1}\right.$, $\left.Y_{o 2}, Y_{o 3}\right)$ for the even-mode excitation of the complementary structure are also related by

$$
\frac{Z_{o i}}{Y_{e i}}=\eta_{o}^{2} / 4, \quad i=1, \cdots, 3
$$

\section{IMPLICATIONS FOR SELF-COMPLEMENTARY STRUCTURES}

This section discusses planar bounded structures that, in addition to satisfying the boundary and symmetry conditions previously described, also are self-complementary. For these self-complementary structures, such as illustrated in Fig. 4, exchanging the metal and open parts of the structure yields an identical (mirror image) structure. Since the structure is identical to its own complement, the results embodied by (19) 
and (20) have some special implications for these bounded symmetrical self-complementary structures.

For a symmetrical self-complementary structure, the oddmode $Z$-parameters are related to the even-mode $Y$-parameters of the same structure by (19). Similarly, the T-circuit impedances of the odd-mode are related to the $\Pi$-circuit admittances of the even-mode of the same structure by (20). This is useful in modeling because it means that if one can successfully characterize either the odd- or even-mode behavior of a symmetrical self-complementary structure, the characteristics of the other mode can immediately be derived. This property can also be used to verify the consistency of even- and oddmode simulations.

Deschamps showed that for two-port symmetrical selfcomplementary unbounded structures, the components of an impedance matrix representing the relationship between the currents and voltages at the two ports exhibit certain properties [5]. The derivation of these properties depends solely on the duality transformation of the field for the complemented structure, which results in (2) and (3). These equations are identical for unbounded and bounded structures. Therefore, Deschamps' results apply to the bounded symmetrical selfcomplementary structures discussed here. Specifically, for the matrix $Z$ defined by

$$
\left(\begin{array}{l}
V_{1} \\
V_{2}
\end{array}\right)=\left(\begin{array}{ll}
Z_{11} & Z_{12} \\
Z_{21} & Z_{22}
\end{array}\right)\left(\begin{array}{l}
I_{1} \\
I_{2}
\end{array}\right)
$$

where $V_{i}(i=1,2)$ and $I_{i}(i=1,2)$ are the port voltages and currents, defined in Fig. 3, Deschamps showed that

$$
\left\{\begin{array}{l}
Z_{11}=Z_{22} \\
Z_{11}^{2}-Z_{12}^{2}=\eta_{o}^{2} / 4
\end{array}\right.
$$

$Z$ is the impedance matrix of an ideal attenuator having a characteristic impedance of $\eta_{o} / 2 \Omega$. This implies that for the bounded symmetrical self-complementary two-port structures discussed here, terminating one of the antenna ports in $\eta_{o} / 2 \Omega$ will result in an impedance of $\eta_{o} / 2 \Omega$ appearing at the other port. If an active device that is internally matched to $\eta_{o} / 2 \Omega$ at both its input and output is connected to the two ports, both its input and output will be matched to the antenna. This will be truly independent of the details of the antenna structure beyond the symmetry and self-complementary restrictions.

\section{SELF-COMPLEMENTARY GRID AMPLIFIERS}

This section discusses the modeling and design of grid amplifiers with self-complementary horizontally symmetrical unit cells. Consider the self-complementary grid-amplifier configuration shown in Fig. 7. Since the dual of a dielectric is a magnetic material which does not exist in nature, to make the active grid a self-complementary structure, we assume that the grid is suspended in free space. In practice, this is achieved by constructing the grid on a very thin substrate with a low dielectric constant. The input and output polarizers are metallic gratings built on dielectric substrates. The input polarizer metal strips are $\theta^{\circ}$ from the vertical. To keep the input and output signals orthogonal, the output polarizer strips are positioned at an angle of $(\theta-90)^{\circ}$ from the vertical. Unlike the previous

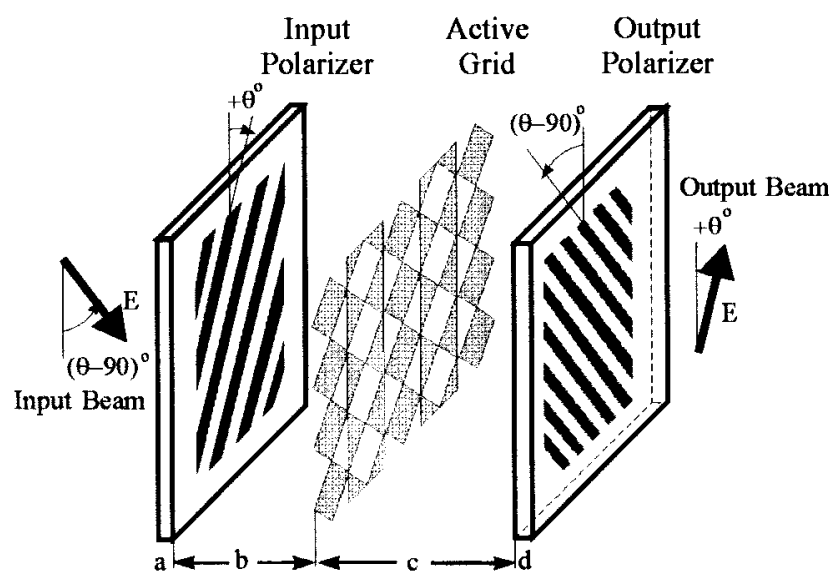

Fig. 7. Perspective view of a self-complementary grid amplifier.

grid amplifiers [1], [2], this design allows the use of single transistors in each unit cell. The input signal that is incident from the left enters the tilted input polarizer normal to its strips (see Fig. 7) and generates RF currents on the input gate leads of the grid. Fig. 8 shows the unit cell and the entire $4 \times 4$ grid amplifier. Currents on the output drain leads generate an output signal that passes through the output polarizer. Similar to the conventional grid amplifiers, the polarizers provide tuning of the amplifier's input and output circuits.

The solution for a self-complementary grid amplifier can be divided into solving for the odd- and even-mode excitations. For each excitation, the current distribution on the grid allows us to define boundary conditions, as shown in Fig. 4(a) and (b). Computer-aided design (CAD) analysis of the unit cell is simpler for the odd-mode excitation. To simulate equivalent-waveguide structures, shown in Fig. 5(a) and (b), it is necessary to excite the internal port 1 shown in these figures. Due to the symmetry of the unit cell, this port can be excited by simulating half of the unit cell and connecting a small piece of a coaxial transmission line to this port. Fig. 9 shows this approach for the odd-mode excitation of the unit cell. This technique is not applicable to the even excitation of the cell because, for the even mode, the waveguide wall at the device port is a magnetic wall and there is no convenient way of inserting a coaxial transmission line at this wall. The three-port waveguide structure, shown in Fig. 9, is used for modeling. Ports 2 and 3 are the front and backside of the grid, and port 1 is the internal port on the grid unit cell where the transistor will be attached. The electric and magnetic walls of the waveguide are shown in this figure. The outer walls of the coaxial transmission line are all electric walls. The coaxial transmission line is deembedded from the final $S$-parameters.

The approach presented here is to analyze the odd-mode excitation of this horizontally symmetrical self-complementary unit cell using Hewlett-Packard's High Frequency Structure Simulator (HFSS). ${ }^{1}$ Due to reciprocity, the $3 \times 3$ scattering matrix resulting from the HFSS simulation can be reduced to a $2 \times 2$ matrix. The port impedance of the combined ports (ports 2 and 3 ) is half the impedance of these ports alone.

\footnotetext{
${ }^{1}$ HP 85180A High-Frequency Structure Simulator, Hewlett-Packard Company, Networks Measurements Division, Santa Rosa, CA.
} 

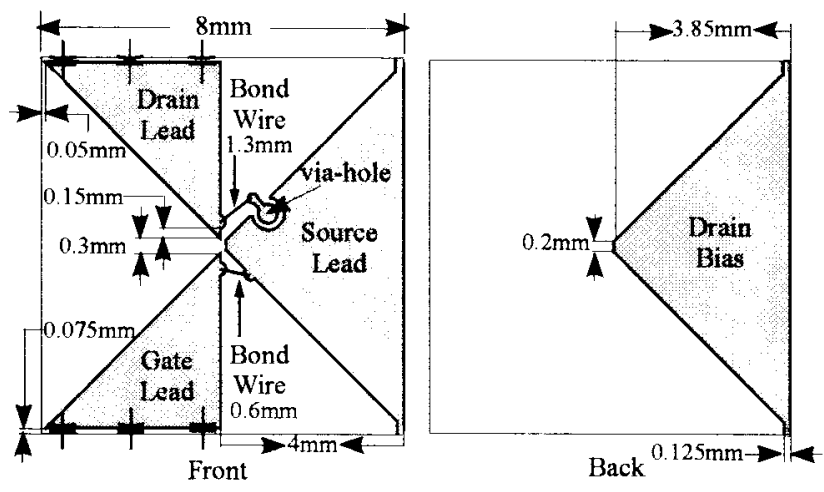

(a)

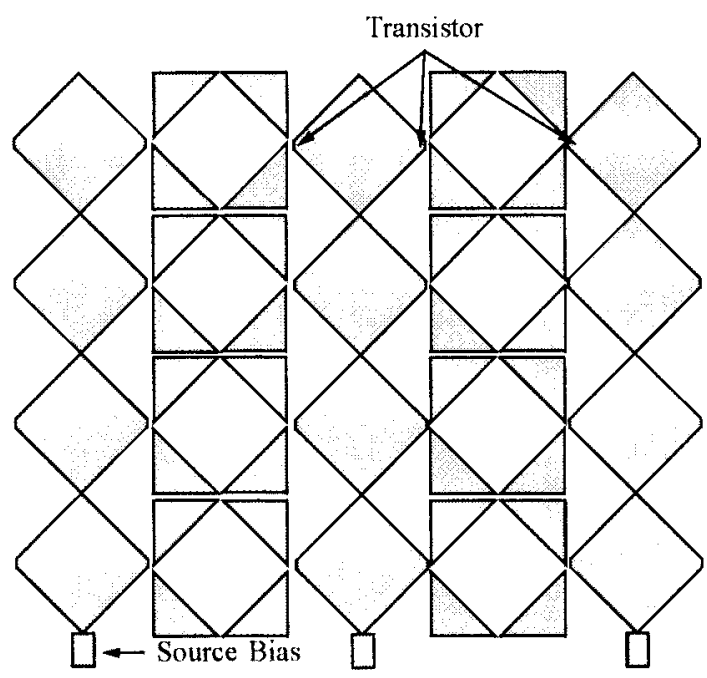

(b)

Fig. 8. (a) Front and backside of the grid amplifier unit cell. (b) Front view of a $4 \times 4$ grid amplifier. The drains are biased from the back side. The drain diamonds are behind the source diamonds.

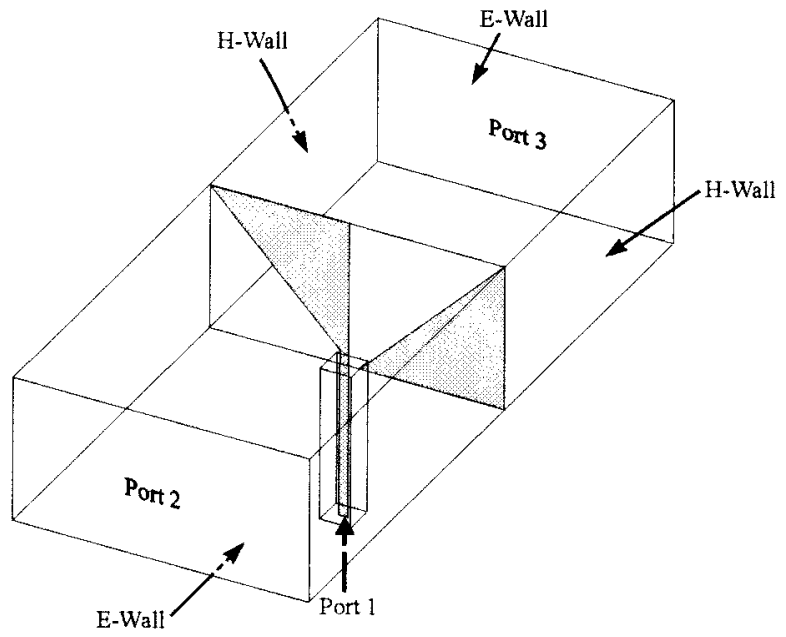

Fig. 9. Layout of the HFSS half-cell used in simulating the odd-mode excitation of the grid unit cell. The coaxial stub used to get access to the internal port of the grid is deembedded from the final $S$-parameter file.

The $Z$-parameters of this structure can be calculated from the $S$-parameters derived from the HFSS simulation. These $Z$-parameters are used to calculate the impedances of the components in the T-equivalent circuit, as shown in Fig. 6(a).

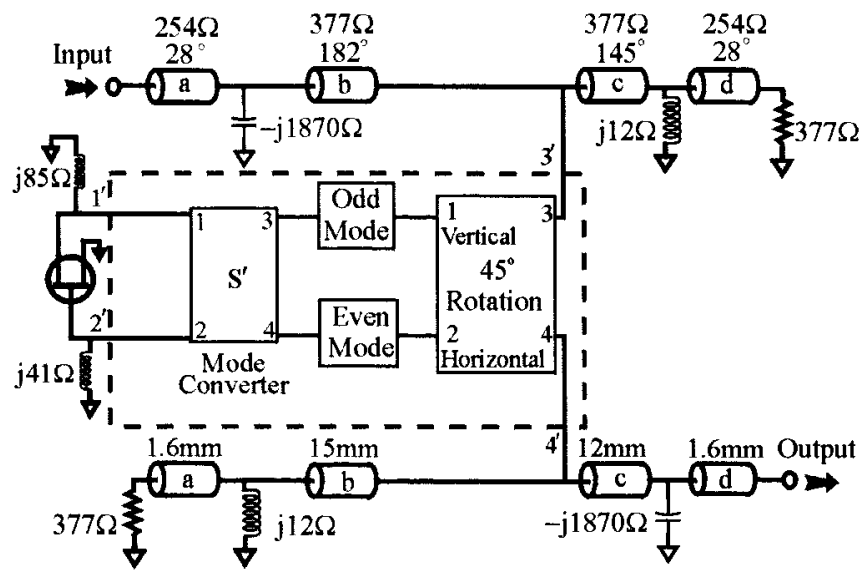

Fig. 10. The equivalent-circuit model of the grid in Fig. 7 at $10 \mathrm{GHz}$.

Note that the full-cell T-circuit has impedances twice that of the half-cell equivalent circuit. The admittances of the full-cell $\Pi$-equivalent circuit, shown in Fig. 6(b), are derived using (20) and the impedances of the T-circuit. By combining the oddand even-mode equivalent circuits, the model for the entire cell can be derived.

The grid shown in Fig. 7 is built on a 10-mil-thick Rogers Duroid substrate with a relative dielectric constant of 2.2. The unit cell, shown in Fig. 8(a), is $8 \mathrm{~mm}$ on a side. The input beam is coupled to the gate of the transistor through the gate lead and the output is radiated from the drain lead, orthogonal to the input. In order to provide bias to the transistors, a doublesided design is used. The source leads in each column are connected to each other and biased from the front of the grid. A wire bond connects each drain lead to a via-hole that is connected to the backside of the grid. The drain bias leads in one column are connected to each other and biased at the backside of the grid. Each gate is shorted to the source through a bond wire. The inductance of the gate and drain bond wires are important in the design of the grid and will be discussed later. To dc isolate the gate of one cell from the drain of the cell below, a $0.15-\mathrm{mm}$ slot is used to separate the two. Three 20-pF chip capacitors, shown in Fig. 8(a), are used to make an RF connection between the gate and drain of neighboring cells. The transistors are Fujitsu FLK012XP MESFET's. Fig. 8(b) shows how the unit cells are connected to construct a $4 \times 4$ grid and does not include the details of each cell. Each arrow in this figure points to the location of the transistor in a unit cell. The transistors are glued on the source lead, and connections to the gate, drain, and source are made with wire bonds.

Fig. 10 shows the transmission-line equivalent circuit for the amplifier configuration of Fig. 7 and unit cell of Fig. 8(a). The dimensions of $a, b, c$, and $d$ correspond to those used for designing the prototype amplifier. Free space for this square unit cell is represented by a resistor with a characteristic impedance of $\eta_{0}=377 \Omega$. The dielectric substrates and air gaps between them are modeled as transmission lines [2]. Polarizers are modeled as inductors or capacitors depending on the polarization. To model the transistor, the $S$-parameters of the FET are normalized to $377 \Omega$. A rotation transformation matrix is defined to separate the incoming signal to its vertical 
and horizontal components, and also to combine the vertical and horizontal components of the outgoing signal to form the output. The four ports of this rotation matrix are the vertical and horizontal polarizations for ports 1 and 2 , and the input and output signals to the grid amplifier for ports 3 and 4 . The $S$-matrix is defined by

$$
S=\left(\begin{array}{cccc}
0 & 0 & -\sin \theta & \cos \theta \\
0 & 0 & \cos \theta & \sin \theta \\
-\sin \theta & \cos \theta & 0 & 0 \\
\cos \theta & \sin \theta & 0 & 0
\end{array}\right)
$$

where $\theta$ is the output-signal angle from the vertical, as shown is Fig. 7.

The vertical component of the input signal is connected to the waveguide port of the T-equivalent circuit of the unit cell, and the horizontal component of the input excites the waveguide port of the $\Pi$-equivalent circuit. The even- and odd-mode device ports are then related to the physical device ports, shown in Fig. 3, using the mode converter $S^{\prime}$ described below.

Define ports 1 and 2 corresponding to voltages 1 and 2 in Fig. 3, and define port 3 as the odd-mode device port [port 1 in Fig. 5(a)], and port 4 as the even-mode device port [port 1 in Fig. 5(b)]. Equations (4)-(7) that relate $V_{1}, V_{2}, V_{o}, V_{e}$ can then be expressed in terms of a mode-conversion matrix

$$
S^{\prime}=\left(\begin{array}{cccc}
0 & 0 & 1 / 2 & 1 \\
0 & 0 & -1 / 2 & 1 \\
1 & -1 & 0 & 0 \\
1 / 2 & 1 / 2 & 0 & 0
\end{array}\right)
$$

The dashed rectangle in Fig. 10 is the passive metal structure of the amplifier unit cell discounting the bond wires shown in Fig. 8(a). The input signal enters port $3^{\prime}$ and the output exits from $4^{\prime}$. Port $2^{\prime}$ is connected to the gate of the transistor and the drain is attached to $1^{\prime}$. For the amplifier to work properly, the transmission coefficients from the input to the gate $\left(S_{2^{\prime} 3^{\prime}}\right)$ and the drain to the output $\left(S_{4^{\prime} 1^{\prime}}\right)$ have to be large, and the transmission coefficients from the input to the drain $\left(S_{1^{\prime} 3^{\prime}}\right)$ and gate to the output $\left(S_{4^{\prime} 2^{\prime}}\right)$ have to be small. Resistive loads of $\eta_{o} / 2 \Omega$ at the device ports $1^{\prime}$ and $2^{\prime}$ are found to be the optimum loads for accomplishing this, as shown in Section IV. Therefore, bond wires at the gate and the drain of the FET are used to resonate with the input and output capacitance of the FET and present a resistive load at ports $1^{\prime}$ and $2^{\prime}$ of the unit cell. The measured inductance of a bond wire with a diameter of $0.7 \mathrm{mil}$ is $1 \mathrm{nH} / \mathrm{mm}$. The required inductance of 1.3 and 0.6 $\mathrm{nH}$ is achieved by adding 1.3- and 0.6- $\mathrm{mm}$ bond wires at the drain and gate of the unit cell, respectively. Different angles for the incoming signal were also considered. An input beam with an angle of $45^{\circ}$ from the vertical is the optimum. The theoretical predicted gain for the circuit in Fig. 10 is $14 \mathrm{~dB}$ at $8 \mathrm{GHz}$.

\section{Gain Measurement}

The small-signal gain of this grid was measured by placing the grid in the far field of two cross-polarized horns [1], [2]. The amplifier did not show significant gain with the polarizers positioned as shown in Fig. 10. We suspect that the reason

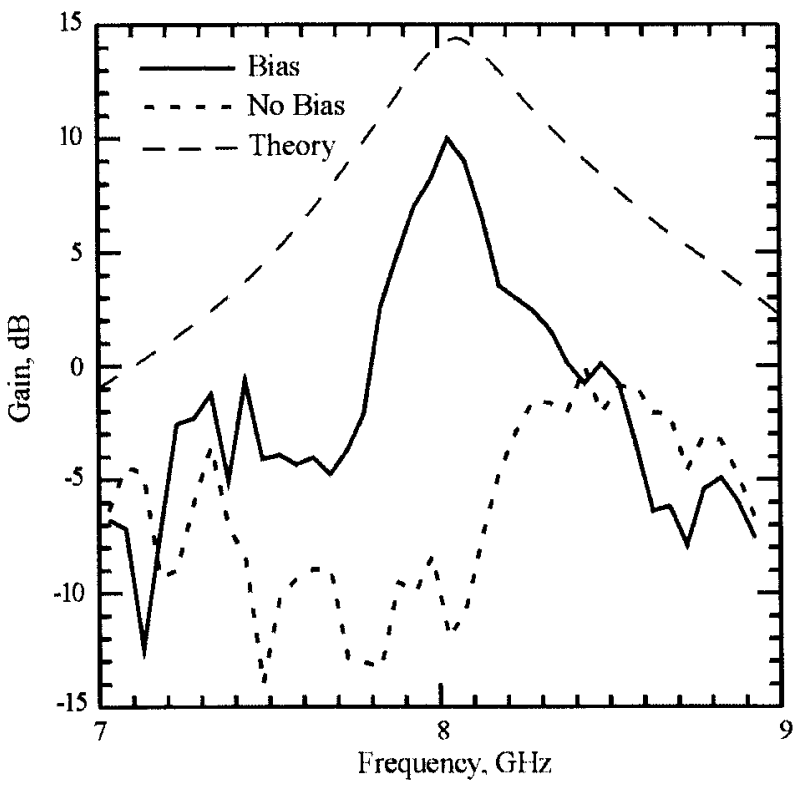

Fig. 11. Gain response of the amplifier grid. The theory is for the grid assembly of Fig. 10. For the measured gain, the output polarizer is 1-2 mm away from the grid and the input polarizer is at a distance of $2.8 \mathrm{~cm}$ from the grid instead of $c=1.2 \mathrm{~cm}$ and $b=1.5 \mathrm{~cm}$ in Fig. 10. Also, tuning slabs are used in the measurement.

might be in part due to the use of a simpler structure in HFSS modeling of the unit cell. A unit cell similar to Fig. 9 was used because HFSS was unable to simulate the more complicated unit cell of Fig. 8(a). However, with the output polarizer 1-2 $\mathrm{mm}$ away from the grid (instead of $c=1.2 \mathrm{~cm}$ in Fig. 10) and the input polarizer $2.8 \mathrm{~cm}$ from the grid (instead of $b=1.5 \mathrm{~cm}$ in Fig. 10), we measured the gain curve shown in Fig. 11. In addition, for this measurement, tuning slabs with a relative dielectric constant of 2.2 were used at the input and output. The input tuning slab is $1 \mathrm{~cm}$ away from the input polarizer and the output tuning slab is $1.8 \mathrm{~cm}$ away from the output polarizer. This gain is measured for a drain voltage of $1.85 \mathrm{~V}$ and a total drain current of $850 \mathrm{~mA}$. The measured peak gain is $10 \mathrm{~dB}$ at $8 \mathrm{GHz}$ and the $3-\mathrm{dB}$ bandwidth is 210 MHz. The gain of the amplifier when it is not biased is also shown in Fig. 11. At peak gain, the difference between the biased and unbiased gain is over $20 \mathrm{~dB}$. To insure that the grid is stable at this bias, the output power was measured versus the input power. A linear dependence between the input and output power indicates that the grid is stable.

As described earlier, tuning for maximum gain, shown in Fig. 11, requires that the output polarizer be placed very close to the grid amplifier. This does not satisfy the assumptions inherent in the simple transmission-line model, shown in Fig. 10, which only considers the propagating TEM mode [6]. Therefore, a theoretical gain curve for our measurement using a transmission-line equivalent model is not possible. However, for comparison purposes, Fig. 11 also shows the theoretical curve of the grid assembly, shown in Fig. 10. With the newer versions of HFSS and the increased computing power available, we hope to have better results in the future by simulating the unit cell with all its details [see Fig. 8(a)] instead of the simplified version of the cell (see Fig. 9). 
The grid suffered from intermittent oscillations at higher drain voltages. The onset of these oscillations were at a drain bias voltage above $1.85 \mathrm{~V}$ and a bias current above $850 \mathrm{~mA}$. The oscillation frequency and power were a function of both bias voltage and time. These oscillations covered a range of frequencies around $8.5 \mathrm{GHz}$.

\section{CONCLUSIONS}

An extension to Deschamps' theorem for three-terminal horizontally symmetrical complementary bounded structures is presented. This theorem is used to design a selfcomplementary grid amplifier. The amplifier has a gain of $10 \mathrm{~dB}$ at $8 \mathrm{GHz}$. The stability of the amplifier is a problem and is an area of future investigation. It may also be fruitful to investigate the use of the devices having broad-band internal matching at both input and output to a real impedance of $\eta_{o} / 2 \Omega$ since such devices would then have a broad-band match to the antenna structure.

\section{REFERENCES}

[1] M. Kim, E. A. Sovero, J. B. Hacker, M. P. De Lisio, J.-C. Chiao, S.-J Li, D. R. Gagnon, J. J. Rosenberg, and D. B. Rutledge, "A 100-element HBT grid amplifier," IEEE Trans. Microwave Theory Tech., vol. 41, pp. 1762-1771, Oct. 1993

[2] M. P. De Lisio, S. W. Duncan, D.-W. Tu, C.-M. Liu, A. Moussessian, J. J. Rosenberg, and D. B. Rutledge, "Modeling and performance of a 100-element pHEMT grid amplifier," IEEE Trans. Microwave Theory Tech., vol. 44, pp. 2136-2144, Dec. 1996.

[3] R. M. Weikle, Quasi-Optical Planar Grids for Microwave and Millimeter-Wave Power Combining, Ph.D. dissertation, Dept. Elect. Eng., California Inst. Technol., Pasadena, CA, 1992.

[4] H. G. Booker, "Slot aerials and their relation to complementary wire aerials (Babinet's Principal)," J. Inst. Elect. Eng., pt. III-A, pp. 620-627, May 1946.

[5] G. A. Deschamps, "Impedance properties of complementary multiterminal planar structures," IRE Trans. Antennas Propagat., vol. AP-7, pp. 371-378, Dec. 1959.

[6] J.-C. Chiao, J. Bae, K. Mizuno, and D. B. Rutledge, "Metal mesh couplers using evanescent waves at millimeter and submillimeter wavelengths," in 19th Int. Conf. Infrared Millimeter-Wave, Sendai, Japan, Oct. 1994, pp. 365-366.

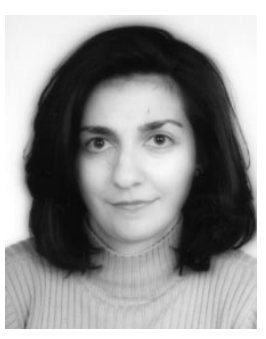

Alina Moussessian (S'97-M'98) was born in Tehran, Iran, on June 21, 1966. She received the B.S. degree in electrical engineering from the Iran University of Science and Technology, Tehran, Iran, in 1988, and the M.S. and Ph.D. degrees in electrical engineering from the California Institute of Technology, Pasadena, in 1992, and 1997, respectively.

In June 1997, she joined the Radar Science and Engineering Section, Jet Propulsion Laboratory, Pasadena, CA. Her research interests include microwave, millimeter-wave and submillimeter-wave power combining, beam-steering, and computer-aided design and measurement of microwave circuits.

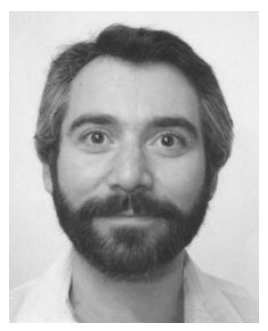

James J. Rosenberg (M'89) received the Sc.B degree in engineering from Brown University, Providence, RI, in 1979, the M.S. degree in electrical engineering from the University of California at Berkeley, in 1980, and the Ph.D. degree from Columbia University, New York, NY, in 1983.

From 1983 to 1990, he was an Assistant and Associate Professor of engineering at Brown University. From 1990 to 1991, he was the Deputy Manager of the Microdevices Laboratory, NASA Jet Propulsion Laboratory, Pasadena, CA. From 1991 to 1992, he served as the Director of Engineering for the Germanium Power Devices Corporation, a manufacturer of semiconductor devices. Since 1992, he has been with Harvey Mudd College, Claremont, CA, where he is currently an Associate Professor. His research interests include semiconductor devices, analog and digital signal-processing circuits, microwave amplification systems, and clock synchronization schemes for high-speed digital systems.

Dr. Rosenberg is a member of Tau Beta Pi. He was the recipient of the NSF Fellowship for graduate study, NSF Presidential Young Investigator Award, and the 1988 Technical Analysis Corporation President's Award for Excellence in Teaching.

David B. Rutledge (S'77-M'77-SM'89-F'93), for biography, see this issue, p. 1955. 